\title{
Long-term correlations in the surface behavior of dolphins
}

\author{
R. Ferrer i CANChO ${ }^{1,2}$ and D. Lusseau ${ }^{3,4}$ \\ 1 Dip. di Fisica. Università "La Sapienza". Piazzale A. Moro 5. 00185 Roma, Italy. \\ 2 Present address: Departament de Física Fonamental, Universitat de Barcelona. Martí \\ i Franquès 1, 08028 Barcelona, Spain. \\ 3 University of Aberdeen, School of Biological Sciences, Lighthouse Field Station, George \\ St. Cromarty, Ross-shire, IV11 $8 Y$ J, Scotland, UK. \\ 4 Present address: Dalhousie University, Department of Biology, 1355 Oxford Street, \\ Halifax, NS B3H 4J1, Canada.
}

PACS. 87.23.Ge - Dynamics of social systems.

PACS. 89.90.+n - Other topics in areas of applied and interdisciplinary physics.

PACS. 87.10.+e - General theory and mathematical aspects.

\begin{abstract}
Here we study the sequence of surface behavioral patterns of dolphins (Tursiops sp.) and find long-term correlations. We show that the long-term correlations are not of a trivial nature, i.e. they cannot be explained by the repetition of the same surface behavior many times in a row. Our findings suggest that dolphins have a long collective memory extending back to the 7-th past behavior. As far as we know, this is the first evidence of long-term correlations in the behavior of a non-human species.
\end{abstract}

Increasing levels of approaches can be followed when studying the relationship between consecutive elements within a sequence. The 1st order approach studies elements neglecting preceding elements. The 2nd order approach studies the relationship between an element and the previous one. The $m$-th order approach consists of studying the dependence of one element on the $m-1$ preceding elements. Sequences of behavioral patterns have been studied in many species. The 2nd order approach has been used for birds [1-4], primates [5-7] and cetaceans [5,6,8-12], to cite some examples. As far as we know, the highest orders that have been achieved are the 3rd [5,6] and the 4th [7] in exceptional studies. Although combinations of signals have been the subject of different studies because of their similarity with human phrases or sentences [13,14], a systematic comparison across species using information theory is found only in the pioneering work by McCowan and collaborators $[5,6]$.

Long-range correlations are found in sequences of many different systems, such as the atmosphere [15, 16], DNA [17-22], the human brain [23] and the fossil record [24]. Besides DNA, long-range correlations are found in other symbolic sequences. Of special interest here are literary texts [25-29] and other sequences of human behavior [30], since they constitute, as far as we know, the only evidence of long-distance correlations in behavioral sequences of a species.

(c) EDP Sciences 
The fact that two behavioral patterns co-occur does not imply, in general, that they are significantly correlated. Statistical physics offers a wide range of measures for detecting longrange correlations. Some examples are Pearson correlation (e.g. [20,31]), information transfer (e.g. [20,31]), Fourier transform (e.g. [20]), wavelet transform (e.g. [32]) or other sophisticated techniques such as detrended fluctuation analysis [33,34]. Here, we chose Shannon's information transfer for three reasons: simplicity, continuity with previous information theory work in animal behavior [5-7] and known advantages over other measures for detecting correlations within symbolic sequences $[31,35]$ (e.g. the ability to detect non-linear correlations). In particular we will focus on two-point correlations between not necessarily consecutive elements of a sequence (e.g. [20,31]). Correlations between blocks of points have been considered by various studies (e.g. $[6,36])$. The goal of the present article is three-fold: (a) going beyond 4 th order approaches and entering the domain of long-term correlations that are found in the vast range of different systems summarized above, (b) providing the first report of long-range correlations in the sequence of behavioral patterns of a non-human species and (c) overcoming the statistical limitations of the methods used in previous animal behavior studies and establishing some guidelines for future studies.

Here we study long-term correlations within sequences of surface behavior of dolphin (Tursiops sp.). Surface behavioral patterns are series of body movements that can be unambiguously identified as a unit. The standard classification of surface behavioral patterns we use here has been used to define the ethogram of a population in many previous studies [37-40]. The ethogram was developed by Schneider after watching the population we studied for more than 1,000 hours [41]. For instance, the pattern "tail-stock dive" (TSD) is composed of the following movements: a dolphin surfaces, arches its body above water and increases its angle of re-entrance, only the tail peduncle is lifted out of the water (the tail is not visible above the surface) and the dolphin dives vertically. These behavioral patterns do not represent the entire behavioral repertoire of the population, but are all patterns that always occur at the surface and therefore can always be observed or recorded when performed (the ethogram was censored to ensure that only events that could always been observed were kept for this analysis; the observation point on the boat used for the study was such that other dolphins could not really mask the view of other dolphins).

Little is known about the function of surface dolphin behavioral patterns although it is suspected that some patterns may convey information [8]. On the one hand, aerial behavioral patterns (i.e. jumps) have, for example, been linked to agonistic displays [37,40] and social bound maintenance [42]. On the other hand, percussive patterns (dolphins producing a sound by slapping the water surface with a part of their body) have been shown to convey information about individual's intentions $[8,38,40]$. Many surface behavioral patterns can be observed at the same time step since many dolphins can be performing a surface behavioral pattern at the same time step (non necessarily the same pattern for each individual). Thus, a sequence of surface behavioral pattern consists of a list of bins where each bin contains all the patterns produced by the observed population during a certain time step (Table I). Our data set consists of a collection of 212 sequences recorded between 2000 and 2002 while following bottlenose dolphin (Tursiops sp.) focal schools in Doubtful Sound, Fiordland, New Zealand $[8,43,44]$. The total number of behavioral patterns produced within these sequences is 30,441 . The mean number of patterns per bin is $1.05 \pm 0.54$. The mean elapsed time between one bin and the next is $15.74 \pm 29.08$ seconds. The length of a sequence is theoretically unlimited but in practice it is constrained by the observation period, which cannot exceed one hour to avoid observer fatigue. Thus, we cannot expect to find significant correlations beyond the mean sequence length. The mean sequence length in bins is $136.59 \pm 105.30$.

We define $S=\left\{s_{i}, \ldots, s_{j}, \ldots, s_{n}\right\}$ as the set of surface behavioral patterns, where $n=33$ in 


\begin{tabular}{ll} 
Time step & Patterns \\
\hline$t$ & TSD \\
$t+1$ & TO \\
$t+2$ & AS \\
$t+3$ & FB \\
$t+4$ & TSD \\
$t+5$ & TSD \\
$t+6$ & AS \\
$t+7$ & TSD \\
$t+8$ & AS \\
$t+9$ & LT \\
$t+10$ & LT \\
& LT \\
& LT \\
& LT \\
$t+11$ & LT \\
$t+12$ & AS \\
$t+13$ & VJ \\
$t+14$ & TSD \\
$t+15$ & TSD \\
$t+16$ & TSD \\
$t+17$ & FOB \\
$t+18$ & FOB
\end{tabular}

TABLE I - Sample from a sequence of dolphin surface behavioral patterns starting at time $t$. The fragment has 22 occurrences of patterns in 19 time steps. The actual time elapsed between consecutive time steps is not necessarily the same. Each bin contains all the behavioral patterns produced during a certain time step. There are seven different patterns: TSD (tail-stock dive), TO (tail out), AS (active surfacing), FB (fart blow), LT (lob tail), VJ (vertical jump) and FOB (forced blow). At every time step, only one dolphin in the focal school is performing the behavioral pattern, except at time $t+10$, where there are multiple occurrences of $L T$ at the same time step. That is, all bins, except one, have a single behavioral pattern. In general, when there are multiple patterns at a certain time step, it is possible that one or more individuals produced more than one behavioral pattern. The identity of the producer is not available in our data.

our dolphins. Imagine a sequence $X$ of $T$ bins of patterns performed during the same time step defined as $X=x_{1}, \ldots, x_{i}, \ldots, x_{T}$, where $x_{i} \subseteq S$. We define the temporal distance (in time steps) between patterns in the bin $x_{i}$ and patterns in the bin $x_{j}$ as $|i-j|$, where $|\ldots|$ is the absolute value operator. We define $N_{i j}(d)$ and $p_{i j}(d)$ as the number of times and the proportion of times, respectively, that the pattern $s_{i}$ has at appeared at temporal distance $d$ before the pattern $s_{j}$ within our collection of sequences. We have

$$
p_{i j}(d)=\frac{N_{i j}(d)}{\sum_{s_{i}, s_{j} \in S} N_{i j}(d)} .
$$

We define $p_{i}^{-}(d)$ and $p_{i}^{+}(d)$, respectively, as the proportion of times that $i$ has appeared at temporal distance $d$ before and after any element of $S$ in the collection of sequences. In other words, we have

$$
p_{i}^{-}(d)=\sum_{s_{j} \in S} p_{i j}(d)
$$


and

$$
p_{i}^{+}(d)=\sum_{s_{j} \in S} p_{j i}(d) .
$$

We define the information transfer between patterns at temporal distance $d$ as

$$
I(d)=\sum_{s_{i}, s_{j} \in S} p_{i j}(d) \log \frac{p_{i j}(d)}{p_{i}^{-}(d) p_{j}^{+}(d)} .
$$

Previous studies in animal behavior have not taken into account the fact that finite size effects overestimate the value of the actual correlations $[31,35,45,46]$. Sometimes, the solution adopted in other fields has been to compare the correlations obtained with the those obtained on scrambled sequences [20,47]. This will be the solution we will follow here, where we use $U$ control collections of sequences. Each control collection is obtained by scrambling all the patterns within each of the sequences in the original collection. Here we use simple shuffling (i.e. just a random permutation of the sequence, e.g. [20]). Other kinds of shuffling preserving some statistical properties of the sequence have been considered (e.g. [36]).

Previous studies of animal behavior [5-7] (and also in other fields, e.g. [20,21]) have not considered the fact that some long-range correlations are of a trivial nature. The repetition of the same element many times in a row gives long-range correlations but in that case the relevance of any statistically significant correlation is questionable [19]. In the case of dolphins, it could merely indicate synchronization within the school; for instance, all dolphins diving together. To that aim, we will use two analysis: a standard analysis of correlations and another one filtering out the co-occurrence of an element with itself. The latter is obtained using a slightly modified definition of $I(d)$ where $N_{i i}(d)=0$ is forced when $s_{i}$ has appeared more than once $d$ events before itself. Again, $p_{i j}(d)$ is defined as in Eq. 1. We denote by $I_{0}(d)$ the information transfer when $N_{i i}(d)=0$ is imposed.

Previous animal behavior work has studied the correlation of an element with the $m-$ 1 preceding elements using an information theory approach [5-7]. Here we will study the correlation of an element with elements that appeared $d$ events earlier (i.e. that appeared at distance $d$ before). If $S$ has $n$ elements one has to keep track of the co-occurrence of every pattern with at most $n^{m-1}$ possible combinations of patterns in the first case while in the second case one has to keep track only of the occurrence of every pattern with at most $n$ different patterns. The first way of measuring correlations is more susceptible to finite size effects than the second.

Fig. 1 A-B and C-D show, respectively, that $I(d)$ and $I_{0}(d)$ in real sequences of surface behavioral patterns are significantly higher than the values obtained for scrambled sequences in a long range. We define $d^{*}$ as the maximum value of $d$ where statistically significant correlations are found. We obtained $d^{*}$ approximately as the largest $d$ where the maximum $I(d)$ in $U$ control collections of sequences does not reach the real $I(d)$. We define $d_{0}^{*}$ as the value of $d^{*}$ obtained on $I_{0}(d)$. The probability that $d^{*}$ is wrong due to deficient sampling (i.e. due to not having generated enough control collections of sequences) is smaller than $p=1 / U$. In our case, the null hypothesis is that the real value of $I(d)$ (or $I_{0}(d)$ ) and that of control sequences come from the same distribution. $p$ gives an upper bound for the probability that the null hypothesis is true. In our case, $U=10^{2}$ gives $p=10^{-2}$. We find $d^{*} \approx 9$ and $d_{0}^{*} \approx 7$, providing support for the existence of long-term correlations within the sequences of dolphin surface behavioral patterns with p-value $<10^{-2}$ (if we are more conservative, i.e. considering a very small $\mathrm{p}$-value we get $d^{*} \approx 6$ and $d_{0}^{*} \approx 7$ with $U=10^{4}$ and p-value $\left.<10^{-4}\right)$. 'Long' here does not mean various orders of magnitude, as in DNA studies (e.g. [20]) but a range that has no precedent in animal behavior studies. 

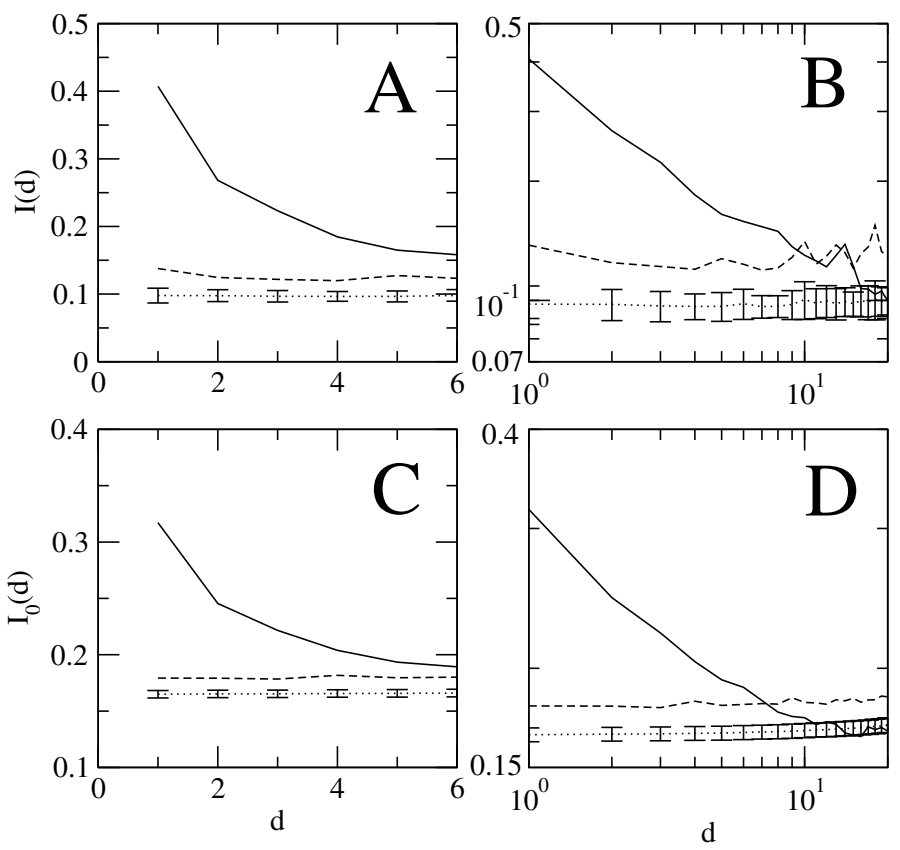

Fig. 1 - A. A detail for short distances of $I(d)$, the information transfer between dolphin surface behavioral patterns at temporal distance $d$. The corresponding mean value over $U=10^{2}$ control collections of sequences is also shown (dotted line). Bars indicate the standard error of the estimated mean. The dashed line indicates the maximum value that $I(d)$ has achieved in the $U$ control collections of sequences. B. The same as A up to approximate point where the real series and the error bars of the control series cross. C. A detail for short distance of $I_{0}(d)$, the information transfer excluding one pattern with itself between dolphin surface behavioral patterns at temporal distance $d$. All series are equivalent to those in $\mathrm{A}$. D. The same as $\mathrm{C}$ up to the approximate point where the real and the error bars of the control series cross. Logarithmic scale in both axes was used in B and D. Natural logarithms were used for the calculation of $I(d)$ and $I_{0}(d)$.

Excluding loops, i.e. co-occurrences of one pattern with itself, may have opposite effects. On the one hand, it eliminates a source of correlations, which alone would result in $I_{0}(d)<$ $I(d)$. On the other hand, excluding loops constrains the set of possible combinations of two patterns at a certain distance, which alone would give $I_{0}(d)>I(d)$. Our results (Fig. 1 C-D) suggest that the span of correlations is not very influenced by excluding loops or not. As far as we know, the only species where long-range correlations within sequences of behavioral patterns have been found are humans [25-30] and the dolphins examined here.

We focused on schools of dolphins and not individuals. In spite of being rougher, group sampling is still useful for understanding the communicative value of behavioral patterns and has been used in humans [48-51] and other primates [52,53]. Patterns could be correlated within and/or between individuals, since we do not have the identity of the producer of each behavioral pattern, The observed correlation could have been the result of a trivial synchronization between members of the school. However, the removal of repetitious patterns did not preclude the appearance of long-range correlation. Our findings suggest that dolphins have a long collective memory extending back to the 7-th past behavioral pattern (we are conservative and choose $d_{0}^{*}$ instead of $d^{*}$ ). The possibility that each individual has a long 
memory of past behavioral patterns cannot be denied and is a challenge for future studies. One should be careful about the interpretation of our results. The fact that dolphin surface patterns at a certain temporal distance are correlated does not mean that patterns are caused by other patterns. Correlation does not imply causation. Correlations between two surface behavioral patterns could be an epiphenomenon of the fact that the two patterns are associated to the same behavioral state or context. We do not intend to explain the nature of the correlations. Besides, further work should be performed to determine if those long-term correlations are a signature of higher order species or a general phenomenon. Our work suggests some guidelines for future studies of animal sequential behavior.

We thank B. McCowan and L. Doyle for helpful discussions. We are very grateful to one anonymous referee for a myriad of suggestion for improving the article. The collection of the data for this study was funded by the New Zealand Whale and Dolphin Trust, Real Journeys Ltd, and the New Zealand Department of Conservation. Additional financial and technical support (DL) was contributed by the University of Otago (Departments of Zoology and Marine Sciences and Bridging Grant scheme). DL thanks his supervisors Liz Slooten and Steve Dawson for their contribution to this research. DL also thanks Susan M. Lusseau and Oliver J. Boisseau for their numerous contributions to this project. This work was also supported by the ECAgents project (RFC), funded by the Future and Emerging Technologies program (ISTFET) of the European Commission under the EU RD contract IST-1940. The information provided is the sole responsibility of the authors and does not reflect the Commission's opinion. The Commission is not responsible for any use that may be made of the data appearing in this publication. It is strictly prohibited to use, to investigate or to develop, in a direct or indirect way, any of the scientific contributions of the author contained in this work by any army or armed group in the world, for military purposes and for any other use which is against human rights or the environment, unless a written consent of all the persons in the world is obtained.

\section{REFERENCES}

[1] M. S. Ficken, E. D. Hailman, and J. P. Hailman, Condor 96, 70 (1994).

[2] J. P. Hailman, Bioacoustics 6, 33 (1994).

[3] J. P. Hailman, M. S. Ficken, and R. Ficken, Ethology 75, 62 (1987).

[4] J. P. Hailman, M. S. Ficken, and R. W. Ficken, Semiotica 56, 121 (1985).

[5] B. McCowan, L. R. Doyle, and S. F. Hanser, Journal of Comparative Psychology 116, 166 (2002).

[6] B. McCowan, S. F. Hanser, and L. R. Doyle, Anim. Behav. 57, 409 (1999).

[7] S. A. Altmann, J. Theor. Biol. 8, 490 (1965).

[8] D. Lusseau, Submitted (2006).

[9] D. P. Nowacek, Behavior 139, 1115 (2002).

[10] V. M. Janik, Science 289, 1355 (2000).

[11] K. Schneider, Behaviour and ecology of bottlenose dolphins in Doubtful Sound, Fiordland, New Zealand, Phd thesis, University of Otago (1999).

[12] E. Slooten, Journal of Mammalogy 75(4), 956 (1994).

[13] C. Crockford and C. Boesch, Behavior 142, 397 (2005).

[14] P. M. Greenfield and E. S. Savage-Rumabaugh, in S. T. Parker and K. R. Gibson, eds., Language and intelligence in monkeys and apes (Cambridge University Press, Cambridge, 1990), pp. 540579 .

[15] R. G. Harrison, J. Atmos. Solar-Terrestrial Physics 66, 1127 (2004). 
[16] A. A. Tsonis, P. J. Roebber, and J. B. Elsner, J. Climate 12, 1534 (1999).

[17] B. Audit, C. Thermes, C. Vaillant, Y. d'Aubenton Carafa, J. Muzy, and A. Arneodo, Phys Rev Lett. 86, 2471 (2001).

[18] W. Li, T. G. Marr, and K. Kaneko, Physica D 75, 392 (1994).

[19] D. Larhammar and C. A. Chatzidimitriou-Dreismann, Nucleic Acids Res. 21, 5167 (1993).

[20] W. Li and K. Kaneko, Europhysics Letters 17(7), 655 (January 1992).

[21] C.-K. Peng, S. V. Buldyrev, A. L. Goldberger, S. Havlin, F. Sciortino, M. Simons, and H. E. Stanley, Nature 356, 168 (1992).

[22] S. V. Buldyrev, A. L. Goldberger, S. Havlin, R. N. Mantegna, M. E. Matsa, C.-K. Peng, M. Simons, and H. E. Stanley, Phys. Rev. E 51, 5084 (1995).

[23] K. Linkenkaer-Hansen, V. V. Nikouline, J. M. Palva, and R. J. Ilmoniemi, Journal of Neuroscience 21, 1350 (2001).

[24] R. V. Solé, S. C. Manrubia, J. Pérez-Mercader, M. Benton, and P. Bak, Adv. Complex Syst. 1, 255 (1998).

[25] M. Montemurro and P. A. Pury, Fractals 10, 451 (2001).

[26] P. K. V. Podgorelec, M. Zorman, T. Kokol, and T. Njivar, Journal of the American Society for Information Science 50, 1295 (2000).

[27] W. Ebeling, T. Pöschel, and K.-F. Albrecht, International Journal of Bifurcation and Chaos 5, 51 (1995).

[28] W. Ebeling and T. Pöschel, Europhysics Letters 26(4), 241 (May 1994).

[29] A. Schenkel, J. Zhang, and Y. Zhang, Fractals 1, 47 (1993).

[30] M. P. Paulus, Phys. Rev. E 55, 3249 (1997).

[31] W. Li, Journal of Statistical Physics 60(5/6), 823 (1990).

[32] A. Arneodo, Y. d'Auberton Carafa, E. Bacry, P. V. Graves, J. F. Muzy, and C. Thermes, Physica D 96, 291 (1996).

[33] C.-K. Peng, S. V. Buldyrev, S. Havlin, M. Simons, H. E. Stanley, and A. L. Goldberger, Phys. Rev. E 49, 1685 (1994).

[34] C.-K. Peng, S. Havlin, H. E. Stanley, and A. L. Goldberger, Chaos 5, 82 (1995).

[35] H. Herzel and I. Große, Physica A 216, 518 (1995).

[36] R. Steuer, W. Ebeling, D. F. Russell, S. Bahar, A. Neiman, and F. Moss, Phys. Rev. E 64, 061911 (2001).

[37] E. Slooten, Journal of Mammalogy 75(4), 956 (1994).

[38] B. Würsig and M. Würsig, Fishery Bulletin 77(2), 399 (1979).

[39] K. Pryor, Report to the International Whaling Commission Special Issue 8, 89 (1986).

[40] D. Herzing, in W. Au, A. Popper, and R. Fay, eds., Hearing by whales and dolphins (Springer, New York, 2000).

[41] K. Schneider, Behaviour and ecology of bottlenose dolphins in Doubtful Sound, Fiordland, New Zealand, Phd thesis, University of Otago (1999).

[42] K.S. Norris, authorB. Würsig,authorR.S. Wells and M. Würsig, The Hawaiian Spinner Dolphin (University of California Press, Berkeley, 1994).

[43] D. Lusseau, K. Schneider, O. J. Boisseau, P. Haase, E. Slooten, and S. M. Dawson, Behavioral Ecology and Sociobiology 54(4), 396 (2003).

[44] D. Lusseau, Conservation Biology 17(6), 1785 (2003).

[45] M. S. Roulston, Physica D 110, 62 (1997).

[46] H. Herzel, A. O. Schmitt, and W. Ebeling, Chaos, Solitons and Fractals 4, 97 (1994).

[47] W. Ebeling and A. Neiman, Physica A 215, 233 (1995).

[48] E. Thomas and T. Malone, Psychological Review 86(4), 331 (1979).

[49] E. Thomas and J. Martin, Psychological Review 83(2), 141 (1976).

[50] J. Gottman, Marital interaction: experimental investigations (Academic Press, New York, 1979).

[51] M. Magnusson, European Journal of Psychological Assessment 12(2), 112 (1996).

[52] P. Haccou, H. Dienske, and E. Meelis, Animal Behaviour 31, 927 (1983).

[53] R. Hinde and J. Stevenson, Advances in the Study of Behavior 2, 267 (1969). 